\title{
Percepción de los maestros sobre las deficiencias visuales y su incidencia escolar
}

\section{Teachers' perception of visual deficiencies and their impact on students}

\author{
Ana FERNÁNDEZ QUEVEDO, José Antonio GARCÍA GARCÍA, Raimundo \\ JIMÉNEZ RODRÍGUEZ y Francisco Javier PERALES PALACIOS \\ Universidad de Granada
}

Recibido: Julio 2014

Evaluado: Octubre 2014

Aceptado: Noviembre 2014

\section{Resumen}

El sentido de la visión constituye la fuente principal de adquisición de información del individuo, de aquí la importancia de un buen diagnóstico y corrección, en su caso, de cualquier anomalía en la misma para un correcto aprendizaje, especialmente en los primeros años de escolarización. En este artículo se exponen los resultados de una encuesta pasada a maestros de centros educativos andaluces mediante la que se pretende poner de manifiesto sus conocimientos sobre las deficiencias visuales que pueden presentar sus alumnos, y la incidencia que podrían tener sobre su rendimiento escolar. Los resultados indican que dicho conocimiento se suele circunscribir a las anomalías de tipo refractivo, considerando que se hallan bien tratadas en sus alumnos. A pesar de la importancia que atribuyen a estas deficiencias sobre el aprendizaje escolar, piensan que otros factores pueden poseer un papel más relevante. Asimismo se consideran partidarios de recibir una formación más completa al respecto.

Palabras clave: deficiente visual, aprendizaje visual, encuesta, enseñanza primaria, formación de maestros.

\begin{abstract}
The sense of vision is people's main source of information acquisition, hence the importance of a right diagnosis and correction, if necessary, of any faults for proper learning, especially in the early years of schooling. This article discusses the results of a survey of teachers in Andalusian schools that aimed at highlighting their knowledge of their students' possible visual deficiencies, and its possible impact on school performance. The results indicate that such knowledge is generally limited to the type of refractive anomalies, and that they think that such anomalies are well treated in their students. Despite the importance they attach to these deficiencies on school learning, they think that other factors may have a greater role. They also consider that better training on this topic is necessary.
\end{abstract}

Keywords: defective vision, visual learning, survey, primary education, teacher training. 
La visión constituye un proceso que integra la información sensorial y motora que involucra más de 20 habilidades y del $65 \%$ de las conexiones cerebrales. Cerca del $80 \%$ de lo que el niño percibe, comprende y recuerda depende de la eficacia del sistema visual (Vergara, 2008). Por tanto, una buena visión en la edad de 6-12 años, esto es, en el ámbito de la Educación Primaria resulta imprescindible para el aprendizaje, puesto que se empiezan a desarrollar actividades tan básicas y necesarias como leer y escribir o las operaciones matemáticas. Una visión deficiente puede ser algo que dificulte notablemente realizar estas actividades.

Determinar en qué medida influye un determinado problema visual en un niño ha sido y es un tema de gran interés ya que siguen existiendo algunas cuestiones pendientes de resolver. Independientemente de qué tipo de problema visual pueda afectar más o en qué pueda afectar, en lo que sí se está de acuerdo es en que los problemas visuales que puedan tener los niños deben ser detectados a tiempo y tratados debidamente, un reto para los educadores y para los ópticos optometristas.

Cuando el niño presenta en sus primeros años dificultades con sus tareas escolares resulta imprescindible una evaluación visual completa, ya que cabe la posibilidad de que padezca un problema visual que pasa desapercibido si solamente se examina la salud ocular y los defectos refractivos. Un bajo rendimiento escolar puede deberse, entre otras causas, a problemas visuales que no han sido diagnosticados (Saona, 2013).

Estamos refiriéndonos a problemas acomodativos, binoculares, oculomotores, la ambliopía, fijación excéntrica o las alteraciones en la visión del color; a pesar de no tratarse habitualmente no son problemas menos importantes que los defectos refractivos. Muchas de las personas cercanas a los niños en edad escolar los desconocen, por lo que si perciben algún síntoma lo pueden achacar a cualquier otra causa, pensando que los problemas refractivos están suficientemente bien tratados.

Los niños no se quejan de una falta de visión porque no saben lo que ello comporta, su visión siempre ha sido así y creen que eso es lo normal. Además, si sus compañeros progresan con normalidad y ellos no, pueden formarse una opinión negativa de sí mismos, desconociendo la causa de ello.

En la mayoría de los centros escolares las revisiones, si se realizan, solo comprueban la agudeza visual, lo que supone un riegso para los alumnos que, inconscientes de su deficiencia visual, seguirán intentando ponerse al nivel de sus compañeros sin mucho éxito. De ahí la importancia de la labor de padres, maestros y ópticos-optometristas u oftalmólogos en la detección precoz de cualquier alteración y remediarla en la medida de lo posible (Mateo, 2010).

Ante esta situación pueden existir distintas alternativas:

- El hacer revisiones completas a todos los niños en esta edad mediante "screenings" visuales no resulta factible, al menos actualmente, por la gran cantidad de tiempo e inversión en material y profesionales para poder llevar a cabo todas las pruebas pertinentes.

- Otra opción sería informar a los padres, algo que por las mismas razones anteriores no puede llevarse a cabo. 
- Por último, nos quedan los maestros, el actuar sobre la comunidad educativa sería la opción más viable por el momento, ya que constituye una población mucho más reducida y, aparte de los padres, los que más tiempo pasan con los niños, sobre todo cuando utilizan su visión cercana, que es el momento en que más problemas visuales se manifiestan. Se hace necesario por tanto que aquellos sean conscientes de los problemas visuales y su importancia.

En este trabajo se pretende dar un primer paso que nos permita aproximarnos a lo que realmente conocen los maestros sobre problemas visuales. Este colectivo puede ser un gran aliado de los profesionales de la visión para resolver los problemas visuales en los niños, ya que conocer el problema es el primer paso para intentar resolverlo.

\section{Algunos precedentes}

La sociedad actual requiere unas demandas visuales cada vez mayores. En las actividades escolares un $90 \%$ de la información que se recibe es visual, llegando hasta un $100 \%$ en las tareas de lectura. En la población infantil, los problemas de aprendizaje alcanzan valores del $11 \%$, estimándose que un $80 \%$ de ellos son debidos a deficiencias de procesamiento visual y un $60 \%$ a trastornos de audición y emocionales (Díaz, Gómez, Jiménez y Martínez, 2004). Los exámenes visuales del colegio (screenings) solo suelen detectar en torno al 5\% (Vergara, 2008) de tales problemas, puesto que se centran en comprobar que existe una buena agudeza visual de lejos para detectar defectos refractivos; esto es importante para tareas como copiar de la pizarra, pero existen otras problemas visuales como los comentados anteriormente, que pueden afectar a tareas como la lectura y escritura de cerca (Garzia et al., 2000).

Algunos ejemplos de signos y síntomas que pueden revelar un posible problema visual son los recogidos en la Tabla 1.

Además de estas disfunciones, cabe nombrar las alteraciones de la visión del color, una anomalía en la que la mayoría de pacientes acaban adaptándose a su condición, ya que no supone un riesgo para su integridad. No obstante, es importante detectarla cuanto antes, porque en los colegios se enseñan muchos conceptos mediante colores y la visión defectiva de éstos puede inducir confusiones y retraso escolar. Aparte, es conveniente informar de que existen una serie de trabajos a los cuales no podrán acceder en su futuro como son las fuerzas armadas, policía, profesiones en el mundo de la electrónica y las telecomunicaciones, topógrafo o los trabajos relacionados con la conducción de vehículos como, por ejemplo, piloto (Mateo, 2010).

Otro estudio realizado por Lázaro (2002) contrastó la incidencia de anomalías visuales con el rendimiento académico. Un grupo de 939 alumnos fueron evaluados por sus maestros en cuanto a su rendimiento académico (bueno, regular, malo o educación especial), y se relacionó con las anomalías oculares que presentaban. Los resultados de este estudio muestran que entre los sujetos que no presentaban anomalía y los que presentaban anomalías refractivas no hay una diferencia significativa en su rendimiento, pero sí que existen diferencias entre los que presentan anomalías 
binoculares, acomodativas y oculomotoras, las cuales estaban asociadas con un menor rendimiento académico.

\begin{tabular}{|c|c|}
\hline Sintomas & Poshleproblema visual \\
\hline $\begin{array}{l}\text { - Se queja de visión borrosa. } \\
\text { - Se frota los ojos frecuentemente. } \\
\text { - Tuerce algún ojo. }\end{array}$ & $\begin{array}{l}\text { Whopia, Hipermetropia o Astigmatismo } \\
\text { (Incapacidad para ver claro en la distancia o de cerca) }\end{array}$ \\
\hline $\begin{array}{l}\text { - Ojo torcido. } \\
\text { - Visión de la profundidad reducida. } \\
\text { - Diferencia entre la claridad de las imágenes. } \\
\text { - Acercarse al papel. }\end{array}$ & $\begin{array}{l}\text { Ambliopia } \\
\text { (Agudeza visual disminuida) }\end{array}$ \\
\hline $\begin{array}{l}\text { - Ojo torcido ("bizco"). } \\
\text { - Supresión de la visión. } \\
\text { - Diplopía. } \\
\text { - Frotarse los ojos. } \\
\text { - Visión de la profundidad reducida. } \\
\text { - Fatiga Visual. } \\
\text { - Cortos periodos de atención. } \\
\text { - Dificultad en la comprensión lectora. } \\
\text { - Evita la tarea de cerca. }\end{array}$ & $\begin{array}{l}\text { Problemas binoculares } \\
\text { (Incapacidad de coordinar los dos ojos) }\end{array}$ \\
\hline $\begin{array}{l}\text { - Se acerca al papel mucho. } \\
\text { - Se queja de visión borrosa. } \\
\text { - Pobre compresión lectora. } \\
\text { - Frotarse los ojos. } \\
\text { - Dice que se le cansan lo ojos. } \\
\text { - Tiene dolores de cabeza cuando lee. }\end{array}$ & $\begin{array}{l}\text { Problemas de enfogue } \\
\text { (Incapacidad para enfocar de forma fácil los ojos o de mantener el } \\
\text { enfoque claro en el tiempo) }\end{array}$ \\
\hline $\begin{array}{l}\text { - Mueve la cabeza excesivamente al leer. } \\
\text { - Pierde el lugar de lectura, se salta líneas al leer. } \\
\text { - Velocidad lectora baja } \\
\text { - Usa el dedo al leer. } \\
\text { - Compresión lectora baja. } \\
\text { - Cortos periodos de atención. } \\
\text { - Omisión de palabras pequeñas al leer. }\end{array}$ & $\begin{array}{l}\text { Problemas de movimientos oculares } \\
\text { (Habilidad inadecuada para mover los ojos de forma suave y precisa al } \\
\text { mirar de un punto a otro) }\end{array}$ \\
\hline $\begin{array}{l}\text { - Confunde palabras que empiezan de forma similar. } \\
\text { - Dificultad para reconocer letras, palabras o formas simples. } \\
\text { - No puede distinguir entra la idea principal y los detalles que no son importantes. } \\
\text { - Problemas para aprender conceptos básicos de matemáticas, tamaño, magritud y posición. }\end{array}$ & $\begin{array}{l}\text { Problemas en la percepción visual de formas } \\
\text { (Incapacidad para discriminar diferencias en tamario o forma) }\end{array}$ \\
\hline $\begin{array}{l}\text { - Problemas para visualizar lo que lee. } \\
\text { - Pobre compresión lectora. } \\
\text { - Problemas con conceptos matemáticos. } \\
\text { - Pobre representación visual del material presentado. }\end{array}$ & $\begin{array}{l}\text { Problemas en la memoria visual } \\
\text { (Incapacidad para recordar y comprender lo que se ha visto) }\end{array}$ \\
\hline $\begin{array}{l}\text { - Escritura y dibujo descuidado. } \\
\text { - No se mantiene sobre la línea. } \\
\text { - Pobre habilidad para copiar. } \\
\text { - Puede responder oralmente pero no prescrito. }\end{array}$ & $\begin{array}{l}\text { Problemas en la integración visuo-motora } \\
\text { (Incapacidad para procesar y reproducir imágenes visuales) }\end{array}$ \\
\hline $\begin{array}{l}\text { - Problemas para aprender derecha e irquierda. } \\
\text { - Invierte letras y palabras. } \\
\text { - Problemas para escrbir y recordar letras y números. }\end{array}$ & $\begin{array}{l}\text { Dificultad con lateralidad y direccionalidad } \\
\text { (Pobre desamollo de la conciencia derechalizquierda) }\end{array}$ \\
\hline
\end{tabular}

Tabla 1. Relación de síntomas con posibles problemas visuales.

Fuente: adaptada de Garzia et al. (2000) y Vergara (2008). 
Tras este estudio, se realizó otro estudio piloto por García, Lázaro y Perales (2013) en el que se estudiaba si los síntomas visuales disminuían tras el tratamiento adecuado. Un total de 52 niños realizaron un entrenamiento visual de entre 7 y 10 sesiones y se evaluaron los síntomas antes y después de este. Los resultados mostraron una mejora de aquellos al final del tratamiento. Es decir, con un tratamiento adecuado se puede mejorar la sintomatología asociada a ciertas anomalías visuales no tratadas, disminuyendo las barreras perceptivas asociadas a tareas como la lectura.

Por otro lado, como se ha visto anteriormente en la Tabla 1, algunos problemas visuales pueden provocar que el niño sea distraído, puesto que le es imposible mantener la atención en una tarea en la que su visión no es buena. Ferrer (1999) afirma que se ha podido comprobar clínicamente una incidencia de alrededor de un $75 \%$ de problemas visuales relacionados con T.D.A. (Trastorno por Déficit de Atención), y cómo aplicando un tratamiento adecuado, estos trastornos han desaparecido en algunos casos y se han atenuado en muchos otros. Muchas de las distintas disfunciones visuales producen síntomas que se pueden confundir con problemas de atención, pero las causas son bien distintas (Vergara, 2008).

A pesar de las evidencias anteriores, no hemos hallado estudios centrados en los propósitos de este artículo más allá de guías para orientadores escolares destinadas a saber cómo abordar la vida escolar para los alumnos con deficiencias visuales.

Es por esto por lo que resulta necesario actuar sobre estos problemas cuanto antes, ya que la mayoría de ellos son tratables y así lograr el máximo rendimiento escolar del niño.

\section{Objetivo}

El objetivo principal de este artículo es el de evaluar el conocimiento de los maestros sobre la optometría y visión en niños de educación primaria, ya que su conocimiento puede ser clave en la detección de problemas visuales y, por tanto, en su solución. Para ello, elaboramos un cuestionario que nos permitiera conocer la situación actual.

Como objetivos específicos iniciales nos planteamos:

1. Tratar de averiguar si los maestros consideran que una buena visión es importante para los niños en su rendimiento escolar, así como hasta qué punto podría influir un problema visual en este.

2. Asimismo, buscamos poner de manifiesto si los maestros son capaces de relacionar síntomas que indican posibles problemas visuales con una mala visión en el niño.

3. También se indagará sobre cuáles son las anomalías visuales que conocen.

4. Por último, trataremos de conocer su opinión con respecto a la información que poseen sobre la visión en los niños y si consideran necesaria un incremento de la misma en su profesión sobre este tema. 
Compararemos los resultados obtenidos en función de los perfiles de los encuestados y la relación entre las respuestas dadas a unas preguntas y otras. Si los resultados obtenidos son relevantes, se valorará la necesidad de realizar un estudio más completo y exhaustivo planteando propuestas de mejora de la encuesta de este estudio piloto.

\section{Metodología}

\section{Descripción de la muestra}

Los centros escogidos han sido de las provincias de Granada y Jaén, intentando elegir una muestra lo más amplia posible con distintos rangos de edad, tipo de colegio y ubicación. En la Tabla 2 se encuentra la relación de los ocho centros participantes en el estudio.

El número total de encuestas válidas fue de 148.La edad media de los encuestados era de 42,2 años, con una experiencia media de unos 18,5 años aproximadamente. Hay más mujeres que hombres en la muestra (68\% frente a un 32\%).

\begin{tabular}{l|l|l|l|l|l}
\hline \multicolumn{2}{c|}{$\begin{array}{c}\text { Provincia de } \\
\text { ubicación }\end{array}$} & \multicolumn{2}{c|}{ Tipo de población } & \multicolumn{2}{c}{ Tipo de centro } \\
\hline Jaén & 4 & Capital & 2 & Público & 5 \\
\hline Granada & 4 & Ciudad media & 2 & Concertado & 3 \\
\cline { 3 - 4 } & & Pueblo & 4 & & \\
\hline
\end{tabular}

Tabla 2. Características de los centros constituyentes del estudio.

\section{Elaboración del cuestionario}

A partir de la bibliografía reseñada anteriormente se han elaborado las preguntas que se han considerado más adecuadas para cumplir con nuestros objetivos, indagando sobre la información que nos interesa conocer. Para el diseño y la redacción del cuestionario se han seguido una serie de recomendaciones generales y recomendaciones específicas válidas para cuestionarios de esta naturaleza (Silva, 2000).

A partir de estos puntos, se comenzó a elaborar un cuestionario que consta de un encabezado y una toma de datos personales y profesionales. En concreto se añadía una breve introducción que decía: "Este formulario tiene como objetivo investigar si el profesorado cree que los problemas visuales en niños de Primaria pueden ser o no una causa de bajo rendimiento escolar. A las preguntas con las respuestas marcadas con $\square$ se puede responder con varias opciones, en el resto marque solo una opción. Por favor, conteste sinceramente.” Posteriormente se les pedía datos sobre:

- Edad, para estudiar su posible influencia en cuanto al conocimiento sobre los problemas visuales.

- Sexo, para la comparativa entre hombres y mujeres. 
- Años durante los que se ha ejercido la profesión, con el mismo fin que la edad.

- Tipo de centro en el que se imparte clase (Público o Concertado), por si existiese alguna diferencia en el conocimiento de los maestros de este tipo de problemas dependiendo del tipo de centro en el que se imparta clase.

- Ubicación del centro, para estudiar si existe diferencia o no entre los maestros de colegios ubicados en pueblos, ciudades o capitales.

Dentro de nuestras posibilidades, se ha intentado recoger la mayor diversidad de perfiles de maestros.

El cuestionario continúa con las siguientes preguntas, en las que se indaga sobre su opinión respecto a cómo están tratados los problemas visuales actualmente, su conocimiento acerca de ellos y si consideran que son importantes para el rendimiento escolar de los niños.

1. ¿Considera que los problemas visuales están debidamente tratados actualmente en nuestra sociedad (España)? Marque la afirmación con la que esté más de acuerdo.

$\square$ Salvo algún caso excepcional, están debidamente tratados

$\square$ Están tratados correctamente los defectos refractivos (miopía, hipermetropía, astigmatismo), pero no los demás

$\square$ Están bien tratados, aunque queda mucho por mejorar

$\square$ Están muy poco tratados

$\square$ No puedo contestar, no tengo información acerca de este tema

Esta primera pregunta trata de averiguar la opinión de los maestros con respecto a cómo se tratan los problemas visuales en España. Hoy en día, la opción más acertada quizá sea la segunda, ya que la mayoría de defectos refractivos están compensados, pero para los demás no se suelen hacer las pruebas pertinentes en los centros ópticos para poder detectarlos.

2. Entre las siguientes opciones, marque las tres que considere que son las principales causas de bajo rendimiento escolar.

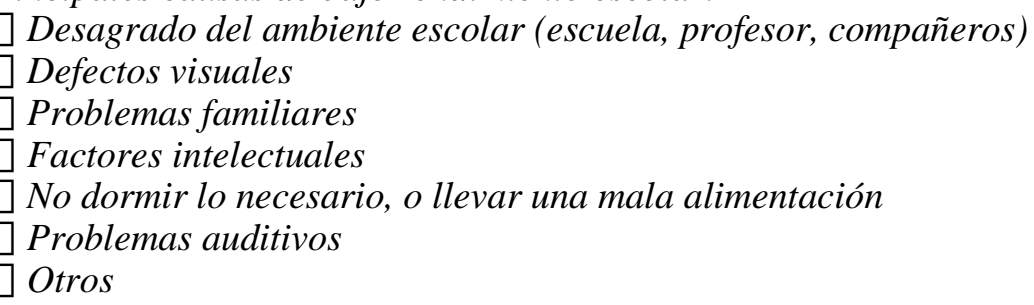

Estos factores se han basado en el trabajo de Hernández (2013). Se pretende conocer si los maestros creen que un defecto visual puede ser una de las principales causas de bajo rendimiento académico. 
3. ¿Cree que una buena visión en el niño es importante para su rendimiento escolar?

$\begin{array}{lllllll} & 1 & 2 & 3 & 4 & 5 & \\ \begin{array}{l}\text { Poco } \\ \text { importante }\end{array} & 0 & 0 & 0 & 0 & 0 & \begin{array}{c}\text { Muy } \\ \text { importante }\end{array}\end{array}$

Se pide que se evalúe la importancia de una buena visión en el niño en relación con lo que puede influir en su rendimiento académico, a fin de conocer si consideran que es un factor importante para el aprendizaje de los niños.

4. De los siguientes, ¿qué tipo de problemas visuales conoce que pueden darse en niños?

$\square$ Miopía, hipermetropía, astigmatismo
$\square$ Problemas acomodativos
$\square$ Problemas binoculares
$\square$ Estrabismo
$\square$ Ojo vago
$\square$ Problemas oculomotores
$\square$ Alteraciones en la visión del color

Se nombran todos los tipos de problemas visuales para que los cuestionados marquen cuáles de ellos conocen. Aunque el estrabismo sea un problema binocular, se ha separado del grupo porque es el que se sospecha que se conoce comúnmente, ya que se puede observar directamente. Esta es una pregunta clave que nos permite saber qué tipos de problemas visuales conocen los maestros, aunque a partir de aquí también pueden ser conscientes de que existen más problemas de los que ellos conocen y esto condicione sus respuestas a las demás preguntas.

5. Evalúe del 1 al 5 (1=poco importante 5=muy importante) la importancia de los siguientes aspectos en la comprensión lectora.

$\square$ Pobreza de vocabulario

$\square$ Problemas de memoria

$\square$ Anomalías en la acomodación visual

$\square$ Escasez de conocimientos previos

$\square$ Anomalías binoculares

$\square$ Inseguridad o falta de confianza

$\square$ Falta de motivación

$\square$ Escasa memoria visual y visualización

Como se ha indicado en la Tabla 1, los problemas binoculares, acomodativos y la escasa memoria visual influyen en la comprensión lectora, además de los demás factores nombrados en este conjunto como la pobreza de vocabulario, que son probables cuando se tienen problemas con esta tarea (González Trujillo, 2005). Con esta evaluación se trata de averiguar si los maestros dan más, igual o menos 
importancia a los problemas visuales que pueden interferir en esta actividad que a los factores a los que normalmente está asociada y que quizá sean más familiares para ellos.

6. De los siguientes síntomas en un niño, ¿qué relacionaría con defectos visuales?

$\square$ Dolor de cabeza
$\square$ Se acerca mucho al papel
$\square$ Es distraído
$\square$ Usa el dedo como guía para leer
$\square$ Ninguno
$\square$ Todos
$\square$ Ns/Nc

El dolor de cabeza, acercarse demasiado al papel, los cortos periodos de atención y usar el dedo como guía para leer son todos síntomas que pueden estar asociados a problemas binoculares, acomodativos u oculomotores (Tabla 1), también pueden asociarse a ambliopía. Se pretende conocer cuáles de estos síntomas son capaces de asociar los maestros a problemas visuales. Además, se analizará el porcentaje de cada respuesta para detectar si los síntomas más reconocidos son los que se relacionan con los problemas visuales más conocidos.

7. Si un niño se frota continuamente los ojos, ¿a qué cree que puede deberse?

$\square$ No le da importancia

$\square$ Tiene algún tipo de alergia

$\square$ Problema visual

$\square$ Tiene sueño

$\square$ Otros

El frotarse los ojos puede ser debido a un problema refractivo, binocular o acomodativo (Garzia et al., 2000), las demás opciones también pueden relacionarse, pero se trata de ver si los maestros conocen que este gesto puede deberse a problemas visuales. Por otro lado, el picor de ojos es uno de los síntomas más característicos de la conjuntivitis alérgica.

Se ha incluido la opción de respuesta "No le da importancia" aunque es de esperar que nadie reconocerá esto, como nos dice Silva (2000) en el punto 6 citado. Esto nos servirá para observar el comportamiento de la muestra que analicemos.

8. Cuando un niño se pierde constantemente de renglón al leer, ¿a qué cree que puede deberse?

$\square$ Dislexia

No ha aprendido a leer con soltura, necesita practicar

$\square$ Problema en la visión

$\square$ Otros 
Perderse de renglón al leer es un síntoma típico de problemas con los movimiento oculares (Garzia et al., 2000). La dislexia también genera este problema (Martínez, 2014), o simplemente una falta de práctica. Al igual que antes, tratamos de ver si esta dificultad se asocia con defectos visuales o es más común asociarlo a otro tipo de problema.

9. Un niño tiene letra irregular y le cuesta escribir en línea recta, usted cree que...

$\square$ Tiene una mala postura y/o coge el lápiz inadecuadamente

$\square$ Aún no tiene soltura

$\square$ Tiene falta de vocabulario

Tiene un problema en la visión

$\square$ Otros

Las principales dificultades de la escritura son sostener y manejar el instrumental adecuadamente, poseer un conocimiento del vocabulario lo más completo posible y haber logrado soltura (Villagrasa, 2012), pero también está relacionado con problemas en la integración visuo-motora (Garzia et al., 2000), y quizá no suela vincularse una letra irregular con este factor. También se relaciona una mala letra con problemas de coordinación ojo-mano, conjuntamente con problemas de acomodación ocular y la deficiente organización espacial debida a problemas de convergencia (Saona, 2005).

10. Cuando un niño guiña uno o los dos ojos para ver la pizarra...

$\square$ Cree que puede presentar miopía

$\square$ No le da importancia

$\square$ Puede presentar ojo vago

$\square$ Cree que es un vicio del niño

Otros

Es muy común que cuando un niño guiña los ojos, los maestros se percaten y cambien el sitio del niño a un lugar más cercano a la pizarra. Con esta pregunta solo se pretende comprobar que este gesto se asocia comúnmente a miopía y quizá sea el problema visual que antes se detecta. Se incluye la opción de ojo vago, que es un problema también muy conocido, pero sin embargo no lleva asociado este gesto de guiñar los ojos.

11. ¿Considera que el profesorado está informado sobre la visión en los niños?

$\square$ Sí

$\square$ No

$\square N s / N C$

12. ¿Considera conveniente la realización de un curso o similar para informarse de este tema?
$\square$ Sí
$\square$ No
$\square N s / N c$ 
Por último, con estas dos cuestiones se pretende saber la opinión de los maestros encuestados respecto a su nivel de conocimiento sobre la visión de los niños y la disposición a realizar un curso sobre esta.

\section{Procedimiento}

Una vez elaborado el cuestionario, se contactó con los directores de distintos colegios, y aquellos que se ofrecieron a colaborar con el estudio lo hicieron llegar a los maestros del centro. Como dijimos anteriormente, el número total de encuestas válidas recogidas fue de 148.

\section{Análisis de datos}

Los datos fueron analizados mediante una estadística descriptiva simple.

\section{Resultados y discusión}

A continuación, se exponen y comentan los resultados derivados de las preguntas realizadas.

En la Figura 1 se muestran las respuestas obtenidas a la primera pregunta.

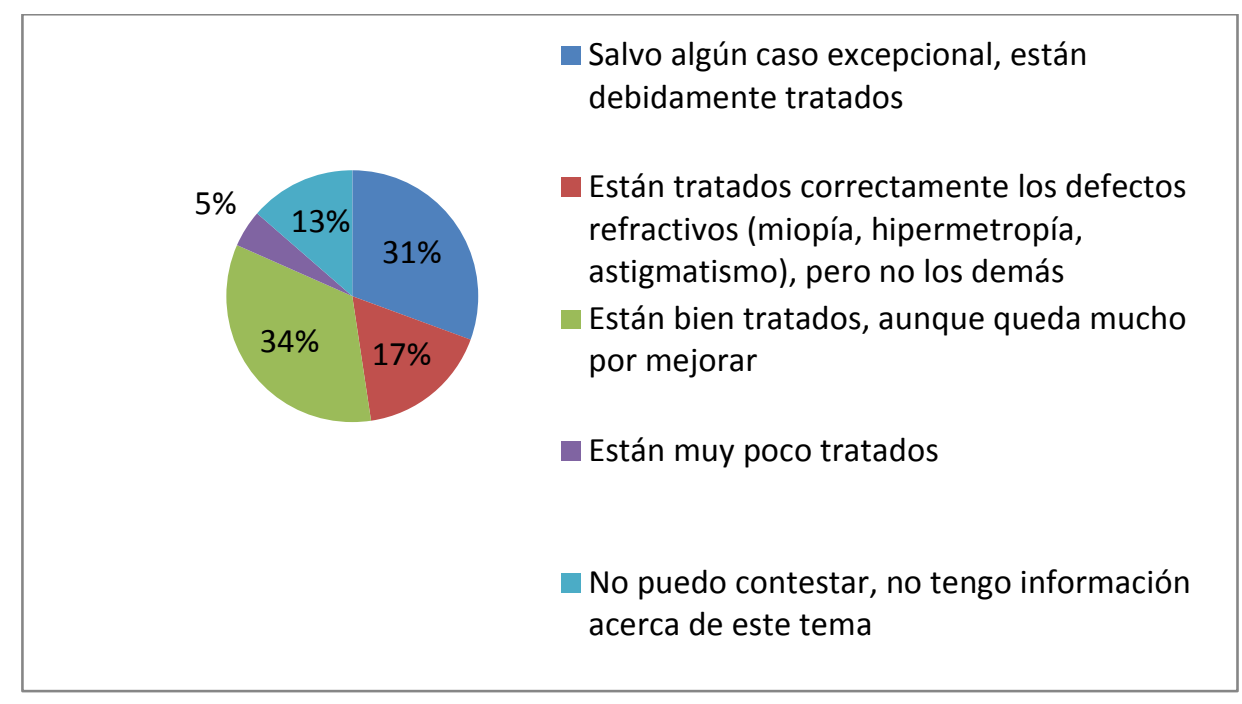

Figura 1. Respuestas obtenidas a la pregunta 1 “¿Considera que los problemas visuales están debidamente tratados actualmente en nuestra sociedad (España)? Marque la afirmación con la que esté más de acuerdo”.

Como podemos observar, la mayoría de los encuestados considera que están debidamente tratados o que existe margen para mejorar su tratamiento. También cabe 
la posibilidad de que no hayan marcado la segunda opción porque no conozcan la existencia de más defectos visuales aparte de los refractivos, por lo que consideran que estos, los únicos existentes a su entender, están bien compensados. Esto hace que nos cuestionemos el porqué de estas respuestas, algo que quizá podamos vislumbrar con el resto de preguntas como, por ejemplo, con las respuestas a la pregunta sobre qué tipo de anomalías visuales conocen.

Los resultados a la cuarta pregunta se muestran en la Figura 2.

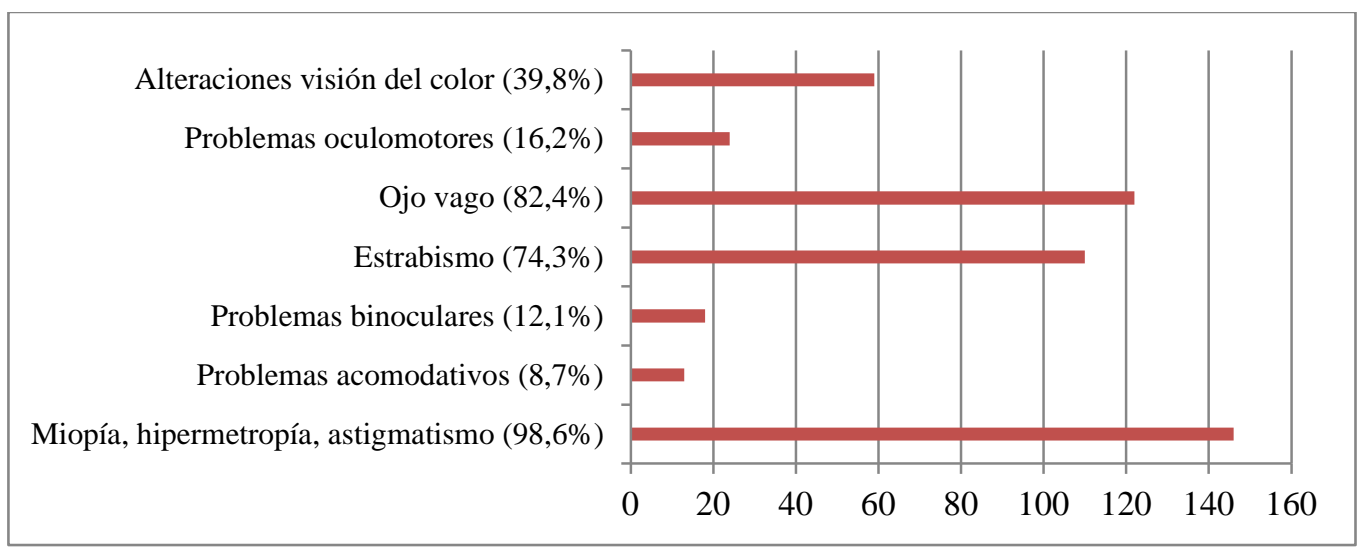

Figura 2. Frecuencias y porcentajes de respuestas a la pregunta 4 "De los siguientes, ¿¿qué tipo de problemas visuales conoce que pueden darse en niños?”.

Como se puede observar, los resultados son sorprendentes ya que realmente sólo conocen los defectos refractivos, lo que explica que en la anterior pregunta se contestase que las anomalías visuales están tratadas correctamente. También hay un gran porcentaje que conoce la existencia de estrabismo y ojo vago pero los demás problemas son desconocidos por la gran mayoría. Además se confirma la sospecha, se conoce el estrabismo pero no los problemas binoculares en sí. Por lo tanto esta es una de las preguntas clave de la encuesta, donde se demuestra el conocimiento de los maestros sobre los distintos problemas visuales existentes.

En la Figura 3 se ha hecho una media de las puntuaciones que se le ha dado a cada factor (quinta pregunta). 


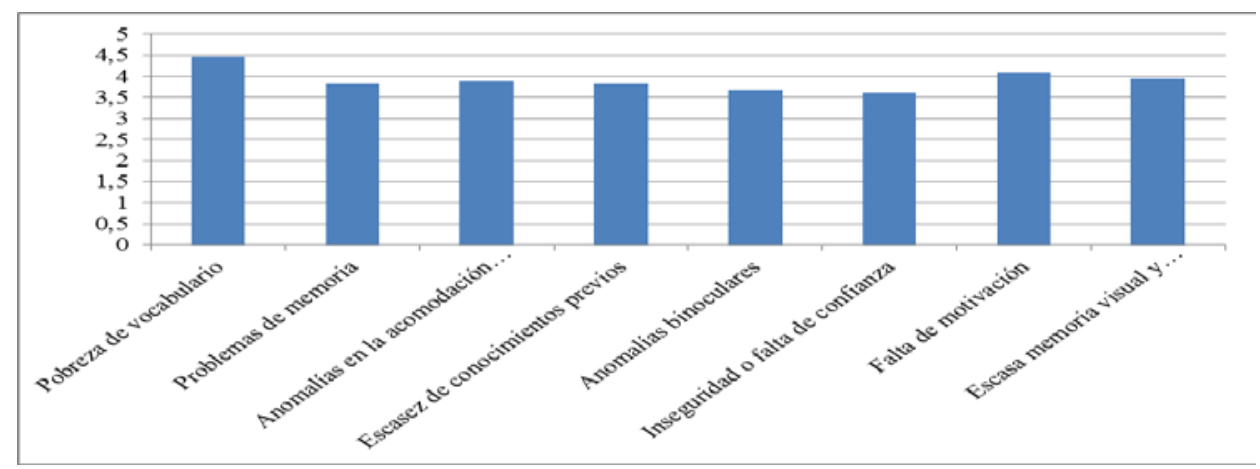

Figura 3. Puntuación media de los factores incluidos en la pregunta 5 "Evalúe del 1 al 5 (1=poco importante 5=muy importante) la importancia de los siguientes aspectos en la comprensión lectora”.

Viendo estos resultados, no podemos decir que no se le dé importancia a los problemas visuales en la comprensión lectora, ya que todos los factores han obtenido un valor parecido y, de una forma u otra, todos influyen según lo que nos dice González (2005) y nos indica la Tabla 1. Podemos reparar en que la opción Ns/Nc es mucho más marcada cuando se pide que se evalúe la influencia de algún tipo de problema visual, aunque puede que a partir de la pregunta anterior sean conscientes de que existen otros problemas que no conocen y esto condicione sus respuestas. Así:

1. Pobreza de vocabulario: $0 \mathrm{Ns} / \mathrm{Nc}$

2. Problemas de memoria: $1 \mathrm{Ns} / \mathrm{Nc}$

3. Anomalías en la acomodación visual: 14 Ns/Nc

4. Escasez de conocimientos previos: $3 \mathrm{Ns} / \mathrm{Nc}$

5. Anomalías binoculares: $32 \mathrm{Ns} / \mathrm{Nc}$

6. Inseguridad o falta de confianza: $1 \mathrm{Ns} / \mathrm{Nc}$

7. Falta de motivación: 4 Ns/Nc

8. Escasa memoria visual y visualización: 6 Ns/Nc

Estos resultados resultan contradictorios si los comparamos con el conocimiento sobre anomalías visuales, ya que solo el 8,7\% conocía las anomalías acomodativas y aquí ha contestado un $90,5 \%$ de los encuestados, al igual que las anomalías binoculares solo las conocía un $12,1 \%$ y ahora ha contestado un $78,3 \%$, ¿ Si no se conocen esos problemas, cómo se puede saber lo que influyen en la comprensión lectora? La respuesta quizá la hallemos en la importancia que le dan los maestros a una buena visión (tercera pregunta), con una media de 4,67 sobre 5 (Figura 4). Es por esto por lo que puede que hayan hecho lo propio respecto a cómo puede influir un problema visual en la comprensión lectora, aunque no sepan muy bien en qué consiste. 


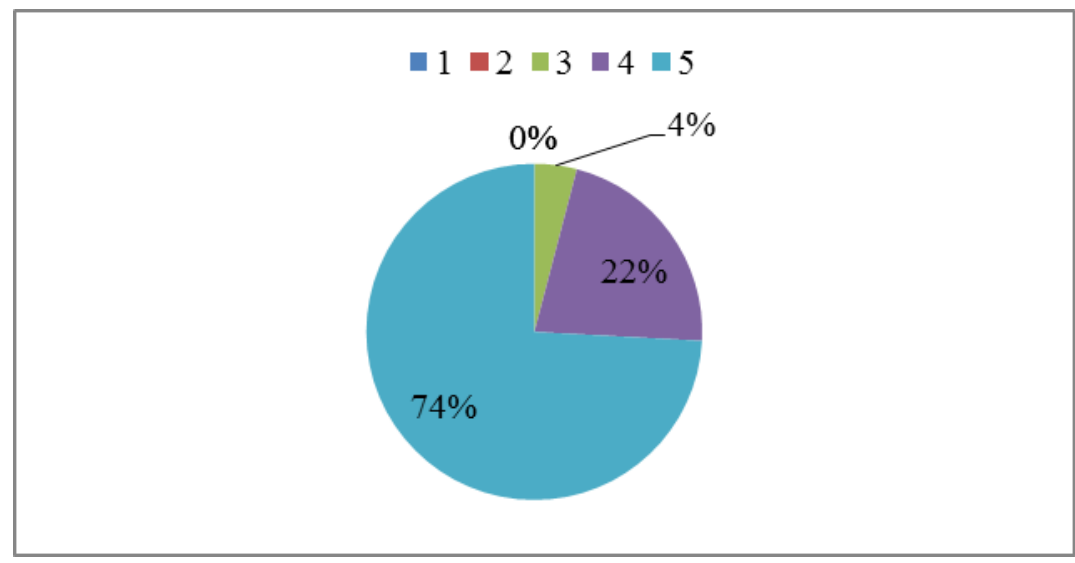

Figura 4. Respuestas a la pregunta 3 “¿Cree que una buena visión en el niño es importante para su rendimiento escolar? (1= Poco importante, 5=Muy importante)”.

Esta es, quizá, una de las preguntas más relevantes de todo el cuestionario. Podemos observar que un 74\% da una puntuación de 5 (Muy importante) a la visión de los niños respecto a lo que influye en su rendimiento académico, sin embargo no conocen muchos de los problemas que pueden darse en aquellos, además de pensar que están solucionados.

La Figura 5 muestra los resultados obtenidos a la segunda pregunta. Lo que sí sabemos es que una mala visión puede influir notablemente en el rendimiento académico y es de los factores menos valorados. En la pregunta anterior $n^{\circ} 3$ sí que se evaluó como muy importante, pero aquí al camuflar la visión con otros factores parece que se le da menos importancia. Los tres factores más marcados fueron: el desagrado del ambiente escolar, problemas familiares y factores intelectuales. Los defectos visuales fueron marcados solo por un $27 \%$ como uno de los tres factores más importantes para el rendimiento escolar del niño.

A continuación se analizarán las preguntas que relacionan síntomas con problemas visuales, donde comprobaremos hasta qué punto son capaces los maestros de asociarlos.

Para la sexta pregunta, en la Figura 6 podemos observar que el síntoma más relacionado con un defecto visual es acercarse mucho al papel, característico de la miopía, uno de los defectos refractivos más conocidos. También puede asociarse a la ambliopía (Tabla 1), que es otro de los defectos visuales más conocidos. Si sumamos el porcentaje de los encuestados que han marcado esta opción y el de los que han marcado la de "todos", obtenemos que un 92,5\% relaciona este gesto con un defecto visual. Si realizamos el mismo procedimiento con el dolor de cabeza, obtenemos un $79,7 \%$ de encuestados que asocian este síntoma a un problema visual. Esto no quiere decir que siempre que un niño tenga cefaleas se deba a un problema visual, pero hay que saber que se trata de un síntoma asociado a la mayoría de problemas visuales, por lo que hay que saber relacionarlo y por ello este porcentaje debería ser aún más elevado. 


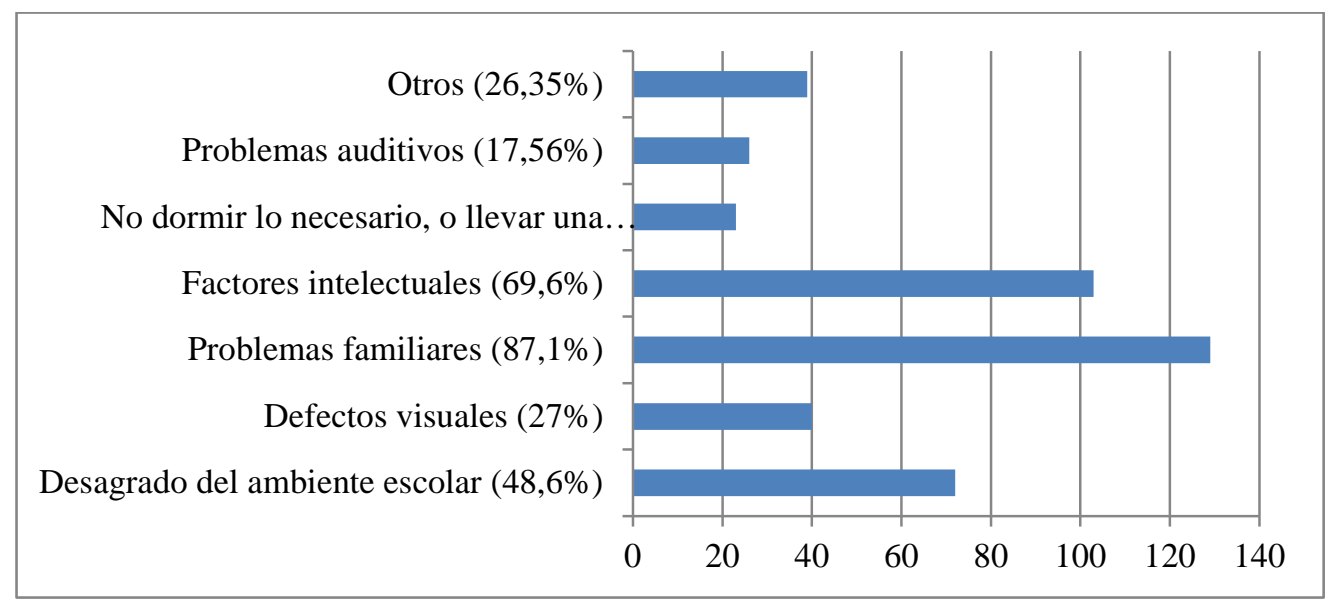

Figura 5. Frecuencias y porcentajes de respuestas a la pregunta 2 "Entre las siguientes opciones, marque las tres que considere que son las principales causas de bajo rendimiento escolar”.

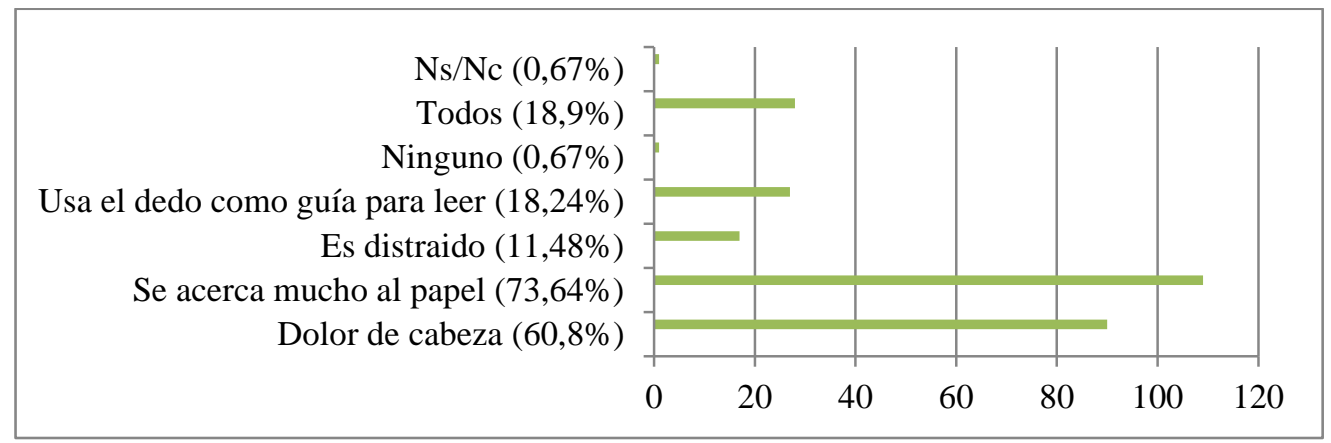

Figura 6. Frecuencias y porcentajes de respuestas a la pregunta 6 "De los siguientes síntomas en un niño, ¿qué relacionaría con defectos visuales?”.

Cuando analizamos los resultados de usar el dedo como guía para leer y la distracción, obtenemos un 37,1\% y un 30,4\%, respectivamente, del total de las respuestas, porcentajes mucho menores que los anteriores. Esto nos indica que las anomalías oculomotoras son menos conocidas ya que síntomas característicos de estas son los cortos periodos de atención y usar el dedo como guía (Tabla 1). La distracción también puede asociarse a anomalías acomodativas o binoculares, ya que al no poder mantener una visión nítida sobre una tarea, el niño deja de fijar su atención sobre esta, distrayéndose fácilmente. De nuevo se pone de manifiesto que los maestros conocen los problemas refractivos y los síntomas de estos, pero no síntomas de otros tipos de anomalías visuales.

En la séptima pregunta se relaciona el frotarse los ojos con problemas visuales; las respuestas obtenidas se recogen en la Figura 7. 


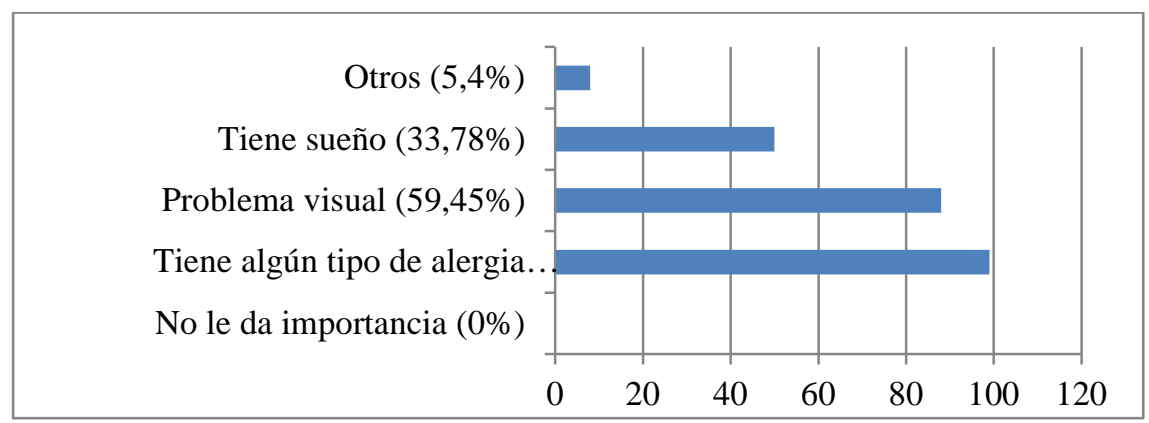

Figura 7. Frecuencias y porcentajes de respuestas a la pregunta 7 "Si un niño se frota continuamente los ojos, ¿̇a qué cree que puede deberse?”.

La opción más escogida es la que dice que puede presentar algún tipo de alergia, por lo que sería lo primero que se pensaría cuando se ve a un niño frotarse los ojos. Esta opción es probable, al igual que todas las que se incluyen, pero también hay que saber relacionarlo con un problema visual, y más de un $40 \%$ no lo asociarían a una anomalía visual, por lo que podría pasar desapercibida. La opción "No le da importancia”, como se preveía, no ha sido escogida por ningún encuestado. Esto confirma una recomendación general para la elaboración de la encuesta (Silva, 2000), relativa a que no deben recogerse opciones que impliquen una valoración por parte del encuestado de ellos mismos, ya que este tiende a responder según la imagen que da. Tal y como indica Silva (2000), aunque es posible que en algún momento un maestro no haya dado importancia a que un niño se frote los ojos, nunca lo reconocerá porque implica una mala imagen de sí mismo.

Continuamos con la octava pregunta. Todas las opciones a esta pregunta pueden ser posibles pero, como podemos ver en la siguiente gráfica de resultados (Figura 8), la opción más marcada es la de un problema visual. Perderse de renglón al leer es un síntoma típico de anomalías oculomotoras, sin embargo, como se ha podido ver anteriormente, solo un 16,2\% (Figura 2) conocía este tipo de problemas y un 37,1\% del total (Figura 6) relacionaba usar el dedo como guía para leer con problemas visuales, otro signo característico de las anomalías oculomotoras.

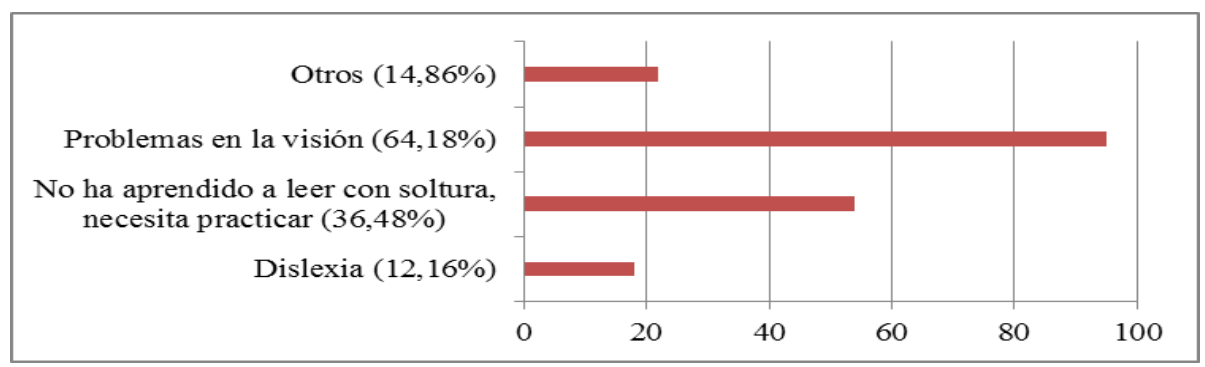

Figura 8. Respuestas a la pregunta 8 “Cuando un niño se pierde constantemente de renglón al leer, ¿̇a qué cree que puede deberse?”. 
Al igual que ocurrió anteriormente en la pregunta 3“¿Cree que una buena visión en el niño es importante para su rendimiento escolar?”, es posible que al ser una encuesta que pretende evaluar el conocimiento de los maestros sobre la visión de los niños y, al formular las preguntas de forma más directa, los encuestados den una mayor importancia a la visión o relacionen síntomas con defectos visuales, aunque puede ser que antes no lo hubiesen pensado.

La novena y décima preguntas tratan de relacionar síntomas con problemas visuales, concretamente una letra irregular con la coordinación ojo-mano y guiñar los ojos con la miopía, respectivamente.

En el primer caso, la opción más marcada es la de un problema visual, sin embargo en esta pregunta la frecuencia de las respuestas es muy similar y no hay ninguna que sobresalga entre las demás. Aun así, esta opción fue marcada por un 43,2\%, lo que nos vuelve a indicar que más de un $50 \%$ de los encuestados no pensaría en asociar un problema visual a una letra irregular o no escribir en línea recta. En la décima pregunta es donde se han obtenido los resultados más inesperados porque sólo el 64,2\% de los encuestados lo relaciona a la miopía, la ametropía que generalmente conoce más la población frente a otras ametropías, pero aun así seguiría existiendo un gran porcentaje de maestros encuestados que no asocian guiñar o cucar los ojos con miopía. Un 47,4\% lo relaciona con ambliopía, sin embargo un niño con ojo vago no guiña el ojo con peor calidad de imagen, puede notar una diferencia de claridad entre imágenes pero es asintomático. El gesto de guiñar un solo un ojo se daría más probablemente en el caso de presentar diplopía por alguna otra causa. Aun así, lo que es de menos importancia es que sepan distinguir entre qué anomalía se manifiesta con unos síntomas y cuál con otros, pero sí que sepan qué tipo de gestos o síntomas puede asociarse a defectos visuales y cuándo deben informar a los padres o tutores para derivar al óptico optometrista u oftalmólogo. De nuevo, se ha incluido la opción "No le da importancia" ocurriendo lo mismo que en la pregunta correspondiente a la Figura 7, donde ningún encuestado ha marcado esa opción, debido a la imagen que daría de sí mismo.

Por último, tenemos las dos preguntas 11 y 12 de opinión sobre la información que poseen los maestros sobre visión en niños y si consideran necesario formarse acerca de este tema, cuyos resultados se muestran en las Figuras 9 y 10. 


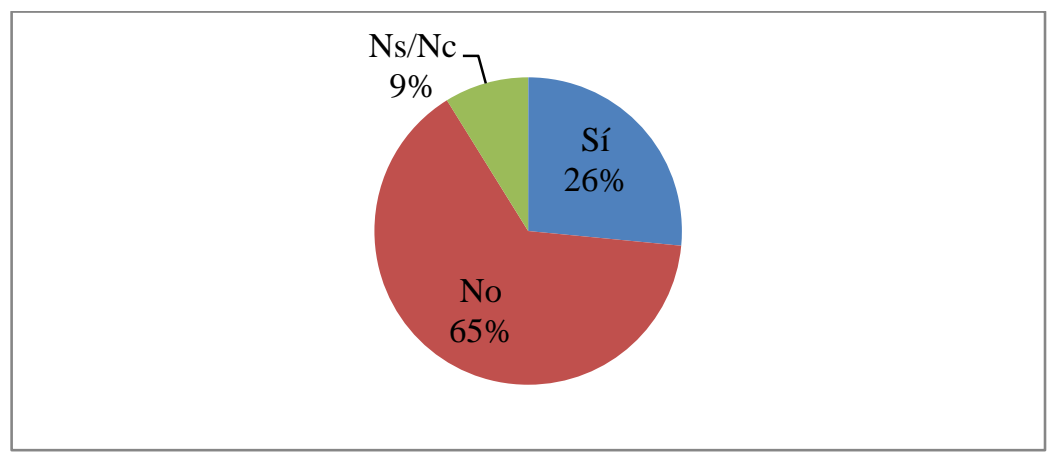

Figura 9. Respuestas a la pregunta 11 “Considera que el profesorado está informado sobre la visión en los niños?”.

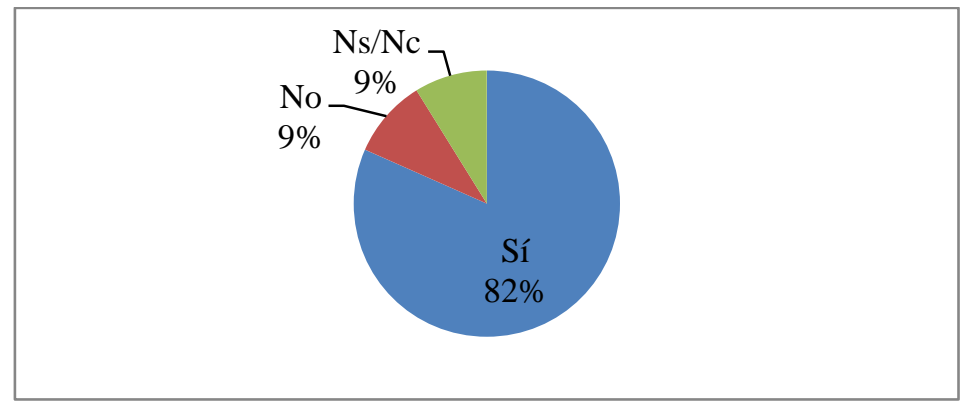

Figura 10. Respuestas a la pregunta 12 ¿Considera conveniente la realización de un curso o similar para informarse de este tema?”.

Son dos preguntas a tener muy en cuenta ya que, como hemos podido ver en los anteriores resultados, parece que los maestros encuestados van siendo conscientes, a medida que rellenan la encuesta, de que existen otros problemas visuales que no conocen y empiezan a reconocer síntomas asociados; por tanto, al llegar a estas dos preguntas de opinión ubicadas al final de la encuesta puede que sean más objetivos con ellos mismos y reconozcan que no están informados sobre el tema y que necesitan más información, como se verá a continuación. Además, a partir de sus respuestas, podremos conocer la predisposición que poseen para ayudar a detectar posibles problemas visuales.

Un 65\% considera no estar informado sobre la visión en los niños, pero aún más elevado es el porcentaje de encuestados que consideran necesaria la formación sobre este tema, un $82 \%$. Viendo esta opinión de los propios maestros, se hace más que necesario hacer algo al respecto. Esto demuestra que los maestros, como buenos profesionales y educadores, se interesan por aquellos aspectos que puedan influir en el rendimiento académico de sus alumnos, y una buena visión es relevante. 
Vamos a analizar estas dos últimas preguntas para observar si las respuestas difieren según el grupo de edad, sexo, experiencia, ubicación o tipo de centro.

En la Tabla 3 se ha calculado el porcentaje de cada grupo de edad que ha contestado que considera no estar informado sobre la visión en los niños y que sí estima necesaria la formación acerca de este tema.

\begin{tabular}{ccc}
\hline Edad & $\begin{array}{c}\text { Considera no } \\
\text { estar } \\
\text { informado }\end{array}$ & $\begin{array}{c}\text { Considera necesaria } \\
\text { la formación }\end{array}$ \\
\hline 20-29 años & $33,3 \%$ & $88,8 \%$ \\
\hline 30-39 años & $62,3 \%$ & $86,9 \%$ \\
\hline 40-49 años & $73,5 \%$ & $82,3 \%$ \\
\hline 50-59 años & $62,8 \%$ & $71,4 \%$ \\
\hline 60-69 años & $77,8 \%$ & $77,8 \%$ \\
\hline
\end{tabular}

Tabla 3. Relación del grupo de edad con la opinión sobre información acerca de la visión y formación acerca de esta.

Los porcentajes obtenidos y mostrados en la tabla son muy parecidos, solo se puede apreciar que hay una evolución en el considerar que no se está informado sobre este tema conforme la edad aumenta. Sin embargo, ocurre todo lo contrario con el interés en recibir información formándose mediante un curso, donde aunque la diferencia de porcentajes no llegue a ser muy significativa, parece que los jóvenes muestran más interés al respecto. Lo mismo sucede con los años de experiencia, ya que normalmente cuanta más edad más años de experiencia, sin embargo no se aprecian diferencias con la tabla anterior.

En cuanto a la variable sexo no se aprecian diferencias de opinión entre hombres y mujeres.

En la Tabla 4 comparamos las respuestas respecto a la ubicación del centro.

\begin{tabular}{ccc}
\hline Ubicación & $\begin{array}{c}\text { Considera no } \\
\text { estar informado }\end{array}$ & $\begin{array}{c}\text { Considera necesaria } \\
\text { la formación }\end{array}$ \\
\hline Pueblo & $56,6 \%$ & $81,9 \%$ \\
\hline Ciudad & $74,5 \%$ & $76,6 \%$ \\
\hline Capital & $72,2 \%$ & $88,9 \%$ \\
\hline
\end{tabular}

Tabla 4. Relación entre la ubicación del centro respecto a la opinión sobre la información acerca de la visión y formación acerca de esta. 
En los centros ubicados en la capital son los que consideran más necesaria la información y es en los pueblos donde un menor porcentaje considera no estar informado, el resto de datos pueden considerarse similares entre los grupos comparados. No se ha realizado comparación entre centros públicos y concertados ya que la opinión no varía significativamente entre un tipo de centro u otro.

En definitiva, lo que sí podemos decir es que los maestros reconocen no estar informados sobre problemas visuales pero muestran una gran predisposición para hacerlo, independientemente de la edad, sexo, experiencia, tipo o ubicación del centro donde trabajen, lo que sugiere que futuras actuaciones deban dirigirse por igual a todo el colectivo.

A continuación, en la Tabla 5, analizaremos si los maestros que han contestado "Sí" a la pregunta “¿Considera que el profesorado está informado de la visión en los niños?”, realmente conocen los distintos tipos de problemas visuales. Del total, son 39 los que han considerado estar informados así que los porcentajes se realizan sobre este número de personas.

\begin{tabular}{lc}
\hline Problema visual & $\begin{array}{l}\text { Lo conoce } \\
\text { un... }\end{array}$ \\
\hline $\begin{array}{l}\text { Miopía, hipermetropía, } \\
\text { astigmatismo }\end{array}$ & $100 \%$ \\
\hline Problemas acomodativos & $5 \%$ \\
\hline Problemas binoculares & $8 \%$ \\
\hline Estrabismo & $77 \%$ \\
\hline Ojo vago & $90 \%$ \\
\hline Problemas oculomotores & $10 \%$ \\
\hline Alteraciones en la visión del color & $33 \%$ \\
\hline
\end{tabular}

Tabla 5. Porcentaje de maestros que han considerado estar informados sobre la visión en los niños y problemas visuales que conocen.

A la luz de estos resultados, vemos que los porcentajes son parecidos a los del total de la muestra encuestada, lo que quiere decir que aunque ellos consideren estar informados sobre la visión en los niños, realmente ello no implica que conozcan los problemas visuales existentes. Al igual que sucede con el total de encuestados, conocen los problemas refractivos, ambliopía y estrabismo, pero no los demás tipos de anomalías.

Comprobemos en la Tabla 6 si estos mismos sujetos saben relacionar síntomas con problemas visuales mediante las respuestas a la pregunta "De los siguientes síntomas en un niño, ¿qué relacionaría con defectos visuales?”: dolor de cabeza; se acerca mucho al papel; es distraído; usa el dedo como guía para leer; ninguno; todos; Ns/Nc. 


\begin{tabular}{lc}
\hline Síntomas & $\begin{array}{l}\text { Lo relacionan con } \\
\text { un problema visual } \\
\text { un... }\end{array}$ \\
\hline Dolor de cabeza & $62 \%$ \\
\hline Se acerca mucho al papel & $74 \%$ \\
\hline Es distraído & $13 \%$ \\
\hline Usa el dedo como guía para leer & $15 \%$ \\
\hline Ninguno & $0 \%$ \\
\hline Todos & $13 \%$ \\
\hline Ns/Nc & $0 \%$ \\
\hline
\end{tabular}

Tabla 6. Porcentaje de maestros que se consideran informados sobre la visión en los niños con los síntomas que relacionan con un problema visual.

De nuevo, como pasaba en los resultados generales, los encuestados saben relacionar con problemas visuales los síntomas típicos de defectos refractivos como acercarse al papel, que puede indicar la presencia de miopía, o el dolor de cabeza, que es un síntoma característico de la mayoría de anomalías. Sin embargo, síntomas típicos de problemas oculomotores como son la distracción y usar el dedo como guía para leer (Tabla 1) solo un pequeño porcentaje lo relaciona con problemas visuales.

Viendo los resultados de estas dos últimas tablas (Tablas 5 y 6 ) podemos decir que, aunque los maestros consideren que tienen información suficiente sobre la visión en los niños, no conocen todos los problemas visuales ni saben relacionar síntomas con estos, por lo que no hay diferencias significativas entre las respuestas de estos y el resto de encuestados que reconocen no estar informados.

La siguiente comparación que podemos realizar es que los sujetos que se consideran informados crean que los problemas visuales están debidamente tratados, por lo que su escasa información es suficiente ya que otros sectores profesionales, como los ópticos optometristas, se ocupan de que estos problemas se solucionen. En la Tabla 7 se muestran los resultados.

\begin{tabular}{lc}
\hline Respuesta & $\begin{array}{l}\text { Está de } \\
\text { acuerdo un... }\end{array}$ \\
\hline $\begin{array}{l}\text { Salvo algún caso excepcional, están debidamente } \\
\text { tratados }\end{array}$ & $31 \%$ \\
\hline $\begin{array}{l}\text { Están tratados correctamente los defectos refractivos } \\
\text { (miopía, hipermetropía, astigmatismo), pero no los } \\
\text { demás }\end{array}$ & $13 \%$ \\
\hline Están bien tratados, aunque queda mucho por mejorar & $46 \%$ \\
\hline Están muy poco tratados & $3 \%$ \\
\hline $\begin{array}{l}\text { No puedo contestar, no tengo información acerca de } \\
\text { este tema }\end{array}$ & $8 \%$ \\
\hline
\end{tabular}

Tabla 7. Porcentaje de maestros que se consideran informados sobre la visión en los niños respecto a su opinión a cómo estos están tratados actualmente. 
La mayoría de respuestas las dan a las opciones "Salvo algún caso excepcional, están debidamente tratados" y a "Están bien tratados, aunque queda mucho por mejorar”, por lo que puede ser que, como consideren que están tratados adecuadamente, se vean exentos de conocer sobre este tema. Aun así, los resultados son muy parecidos a los que obtenemos con el total de encuestados.

Lo que podemos decir a la vista de estos resultados es que las respuestas a las demás cuestiones no cambian si las comparamos con el conjunto total. Este grupo no conoce más tipos de anomalías visuales ni saben relacionar más síntomas con problemas visuales. Se han podido considerar informados por el hecho de que crean que estos problemas están solucionados y a ellos no les incumbe, o porque no sepan de la existencia de más anomalías que las que ellos conocen; no podemos decir que esto ocurra porque no le dan importancia a la visión ya que su valoración en general es de muy importante. Son por tanto resultados extrapolables, independientemente de considerarse informados o no.

\section{Conclusiones}

Tras el análisis de los resultados de la encuesta, podemos enumerar como principales conclusiones las siguientes, relacionándolas con los objetivos específicos señalados inicialmente:

1. Tratar de averiguar si los maestros consideran que una buena visión es importante para los niños en su rendimiento escolar, así como hasta qué punto podría influir un problema visual en este.

El profesorado encuestado concede una gran importancia a una buena visión en los niños para su rendimiento escolar.

Cuando se preguntan posibles causas de un bajo rendimiento escolar, solo un 27\% lo marca como una entre las tres principales causas de bajo rendimiento escolar, dando más importancia a factores intelectuales o problemas familiares. Podríamos decir que las respuestas varían si la pregunta se formula de forma implícita o explícita. Se da más importancia a la visión cuando se pregunta directamente sobre ella.

2. Asimismo, buscamos poner de manifiesto si los maestros son capaces de relacionar síntomas que indican posibles problemas visuales con una mala visión en el niño.

Cuando los maestros conocen un problema visual, como es la miopía o, en algunos casos, los problemas oculomotores, saben identificar sus síntomas. Este hecho proporciona optimismo sobre el papel que pueden jugar aquellos en la detección de las anomalías visuales

3. También se indagará sobre cuáles son las anomalías visuales que conocen.

Los encuestados apenas conocen los distintos problemas visuales existentes, solamente los refractivos y la ambliopía. Al no conocerlos, no saben identificar síntomas típicos de estos. 
Los maestros consideran que las anomalías visuales están suficientemente tratadas actualmente, quizá porque crean que los únicos existentes son los refractivos y dan por supuesto que estos están bien tratados.

A lo largo de la encuesta puede que los maestros se hayan percatado de que existen más problemas visuales que los que ellos conocen, por eso al final un $65 \%$ reconoce no estar informado sobre la visión en los niños. Como se mostró, los que consideran sí estar informados no demuestran poseer mayor conocimiento sobre anomalías visuales que el resto, simplemente puede que desde su desconocimiento acerca de otras anomalías crean que ya está todo tratado adecuadamente, o que consideren que desde su puesto la información que tienen es necesaria porque crean que ya no pueden contribuir más a mejorar la visión de los niños.

4. Por último, trataremos de conocer su opinión con respecto a la información que poseen sobre la visión en los niños y si consideran necesaria un incremento de la misma en su profesión sobre este tema.

Un gran mayoría de los encuestados considera necesaria su formación mediante un curso -o similar- sobre este tema. No existen diferencias entre perfiles de encuestados, solo se aprecia una leve diferencia con la edad y la experiencia; los mayores reconocen su ignorancia acerca del tema mientras los jóvenes creen saber más, siendo estos últimos los que muestran un mayor interés por la formación. En general, los maestros se muestran colaboradores con la detección de anomalías visuales en los niños.

Por consiguiente podemos decir que los maestros pueden constituir un buen aliado del óptico-optometrista u oftalmólogo ya que muestran interés en aprender sobre la visión en los niños y si reconocen los síntomas les resuotará más fácil detectar cualquier anomalía visual que pueda influir en su rendimiento académico. Además, dándonos cuenta a tiempo de una anomalía visual y poniendo remedio mediante el tratamiento más adecuado, los síntomas disminuirán como se mostraba en el estudio de García et al. (2013).

Sería por tanto necesario, desde un punto de vista visual y académico, que se formara a los maestros en el reconocimiento de síntomas en la población escolar, y así poder actuar como primer filtro para detectar a niños cuyo rendimiento académico se pueda ver comprometido.

\section{Referencias bibliográficas}

DÍAZ, S.B., GÓMEZ, A., JIMÉNEZ, C. Y MARTÍNEZ M.P. (2004). Bases optométricas de la lectura eficaz. Madrid: Centro de Optometría Internacional.

FERRER, J. (1999). Los trastornos de la atención y la hiperactividad. Barcelona: Lebon.

GARCÍA, J.A., LÁZARO, M.M. Y PERALES, F.J. (2013). Anomalías de la visión y rendimiento escolar en Educación Primaria. Un estudio piloto en la población granadina. Revista interuniversitaria de formación del Profesorado, 76(27), 101119. 
GARZIA, R.P., BORSTING, E.J., NICHOLSON, S.B., PRESS, L.J., SCHEIMANN, M.M. Y SOLAN, H.A. (2000). Optometric clinical practice guideline. Care of the patient with learning related visual problems. St Louis: American Optometric Association.

GONZÁLEZ TRUJILLO, M.C. (2005). Comprensión lectora en niños: Morfosintaxis y Prosodia en Acción. Tesis Doctoral. Granada: Universidad de Granada,

HERNÁNDEZ, B. (2013). Causas del bajo rendimiento escolar origina un alto nivel de deserción escolar y habilidades para estudiar ayudan a mejorar el rendimiento escolar. Revista Iberoamericana para la Investigación y el Desarrollo Educativo, Julio-Diciembre. Publicación\#11.

LÁZARO, M.M. (2002). Incidencia de las anomalías visuales en niños en edad escolar. Tesis Doctoral. Granada: Universidad de Granada.

MARTÍNEZ, V. (2014). La importancia de la lectura. Dislexia. Centro de especialidades http://www.logopedazaragoza.com/ (Consultado 5 de febrero de 2014).

MATEO, R. (2010). Estudio observacional de la salud visual y auditiva de los alumnos de primaria del colegio la Salle de Mahón. TFM. Barcelona: Universidad Politécnica de Cataluña.

SAONA, C.L. (2005). Importancia del optometrista como profesional de atención primaria y terapeuta visual en la clínica oftalmológica, Institut Oftalmològic Integral, 2 http://www.admiravision.es/ (Consultado 23 de febrero de 2014).

SAONA, C.L. (2013). Visión y aprendizaje en los niños. Blog Admiravisión http://www.admiravision.es/ (Consultado 3 de marzo de 2014).

SILVA, L.C. (2000). La encuesta y el cuestionario. En: Silva, L.C. (ed.). Diseño de muestras y captación de datos para la investigación sanitaria. Madrid: Díaz de Santos S.A, pp. 33-83.

VERGARA, M.P. (2008). Tanta inteligencia tan poco rendimiento. ¿Podría ser la clave para desbloquear su aprendizaje? Albacete: Rona Visión S.L.

VILLAGRASA, M.R. (2012). Disgrafías. Ponencia extraída de la publicación de las "IV Jornadas de Divulgación de la Grafología". Instituto de Estudios Psicografológicos http://www.idauma.com/ (Consultado 7 de febrero de 2014). 


\section{Correspondencia con los autores}

\section{Ana FERNÁNDEZ QUEVEDO}

Facultad de Ciencias. Departamento de Óptica. Edif. Mecenas, desp.145.

Campus Fuentenueva s/n.

Universidad de Granada

18071 Granada

e-mail: anaferq@correo.ugr.es

José Antonio GARCÍA GARCÍA

Facultad de Ciencias. Departamento de Óptica. Edif. Mecenas, desp.145.

Campus Fuentenueva s/n.

Universidad de Granada

18071 Granada

e-mail: jgarcia@ugr.es

Raimundo JIMÉNEZ RODRÍGUEZ

Facultad de Ciencias. Departamento de Óptica. Edif. Mecenas, desp.145.

Campus Fuentenueva s/n.

Universidad de Granada

18071 Granada

e-mail: raimundo@ugr.es

Francisco Javier PERALES PALACIOS

Facultad de Ciencias de la Educación.

Departamento de Didáctica de las Ciencias Experimentales.

Campus Universitario de Cartuja, $\mathrm{s} / \mathrm{n}$.

18071 Granada

e-mail: fperales@ugr.es 